\title{
Cannabidiol use and effectiveness: real- world evidence from a Canadian medical cannabis clinic
}

\author{
Lucile Rapin * D, Rihab Gamaoun, Cynthia El Hage, Maria Fernanda Arboleda and Erin Prosk
}

\begin{abstract}
Background: Cannabidiol (CBD) is a primary component in the cannabis plant; however, in recent years, interest in CBD treatments has outpaced scientific research and regulatory advancement resulting in a confusing landscape of misinformation and unsubstantiated health claims. Within the limited results from randomized controlled trials, and lack of trust in product quality and known clinical guidelines and dosages, real-world evidence (RWE) from countries with robust regulatory frameworks may fill a critical need for patients and healthcare professionals. Despite growing evidence and interest, no real-world data (RWD) studies have yet investigated patients' reports of CBD impact on symptom control in the common expression of pain, anxiety, depression, and poor wellbeing. The objective of this study is to assess the impact of CBD-rich treatment on symptom burden, as measured with a specific symptom assessment scale (ESAS-r).

Methods: This retrospective observational study examined pain, anxiety, depression symptoms, and wellbeing in 279 participants over 18 years old, prescribed with CBD-rich treatment at a network of clinics dedicated to medical cannabis in Quebec, Canada. Data were collected at baseline, 3 (FUP1), and 6 (FUP2) month after treatment initiation. Groups were formed based on symptom severity (mild vs moderate/severe) and based on changes to treatment plan at FUP1 (CBD vs THC:CBD). Two-way mixed ANOVAs were used to assess ESAS-r scores differences between groups and between visits.

Results: All average ESAS-r scores decreased between baseline and FUP1 (all ps < 0.003). The addition of delta-9tetrahydrocannabinol (THC) during the first follow-up had no effect on symptom changes. Patients with moderate/ severe symptoms experienced important improvement at FUP1 (all ps $<0.001$ ), whereas scores on pain, anxiety, and wellbeing of those with mild symptoms actually increased. Differences in ESAS-r scores between FUP1 and FUP2 were not statistically different.
\end{abstract}

Conclusion: This retrospective observational study suggests CBD-rich treatment has a beneficial impact on pain, anxiety, and depression symptoms as well as overall wellbeing only for patients with moderate to severe symptoms; however, no observed effect on mild symptoms. The results of this study contribute to address the myths and misinformation about CBD treatment and demand further investigation.

Keywords: Cannabidiol, Symptoms, Medical cannabis, Effectiveness, Pain, Anxiety, Depression

*Correspondence: Irapin@santecannabis.ca

Research Department, Santé Cannabis, 4150 Ste-Catherine O. Bureau 225,

Montréal, QC H3Z 2Y5, Canada

(c) The Author(s). 2021 Open Access This article is licensed under a Creative Commons Attribution 4.0 International License, which permits use, sharing, adaptation, distribution and reproduction in any medium or format, as long as you give appropriate credit to the original author(s) and the source, provide a link to the Creative Commons licence, and indicate if changes were made. The images or other third party material in this article are included in the article's Creative Commons licence, unless indicated otherwise in a credit line to the material. If material is not included in the article's Creative Commons licence and your intended use is not permitted by statutory regulation or exceeds the permitted use, you will need to obtain permission directly from the copyright holder. To view a copy of this licence, visit http://creativecommons.org/licenses/by/4.0/. 


\section{Background}

Cannabidiol (CBD) is one of the primary cannabinoids found in significant but variable concentrations in cannabinoid-based medicines (CBM). While structurally similar to 49-tetrahydrocannabinol (THC), CBD does not cause intoxication or euphoria (Russo 2017) and has showed considerable tolerability in humans with a low abuse potential (Chesney et al. 2020). This favorable safety profile has led to the recent mitigation of legal and regulatory barriers surrounding purified CBD products in several countries and recent increased interest in CBD treatments. While recent rulings clarified that CBD is not a drug under the 1961 United Nations as Single Convention on Narcotic Drugs, regulatory status in the USA remains extremely confusing. When derived from cannabis, CBD is a schedule 1 drug but when derived from "industrial hemp" plants it may be lawful federally (Corroon and Kight 2018; Corroon et al. 2020). In Canada, CBD is controlled under the Cannabis Act as are all cannabinoids, cannabis, and cannabis-derived products (Canada Go 2021). This regulatory status imparts restrictions and access obstacles for researchers.

CBD is widely touted as a panacea for a wide range of health problems and has been marketed as a dietary and "wellness" product (Russo 2017; Khalsa et al. 2020; Eisenstein 2019). CBD's potential effects as an add-on therapy have been studied for social anxiety disorders, schizophrenia, non-motor symptoms in Parkinson's disease, and substance use disorders (Bergamaschi et al. 2011; Crippa et al. 2019; McGuire et al. 2018; Millar et al. 2019; Prud'homme et al. 2015; Thiele et al. 2019; Leehey et al. 2020). However, the evidence of its effectiveness for indications other than drug-resistant pediatric epilepsy conditions remains very limited (Larsen and Shahinas 2020; Franco et al. 2020) and safety considerations such as drug-drug interactions associated with unsupervised use remain (Chesney et al. 2020; Freeman et al. 2019). Randomized controlled trials (RCTs) are limited in their rigorous design, population sample, and duration of observation making generalization of results and long-term data scarce. Therefore, real-world evidence (RWE) provides valuable insights and supplemental information about the use, safety, and effectiveness of CBD-based treatments (Graham et al. 2020).

RWE from retrospective analyses and patient registries shows that CBMs are used for pain (chronic, neuropathic), mental health conditions, cancer-related symptoms (nausea, fatigue, weakness), HIV/AIDS, and neurological conditions (Bonn-Miller et al. 2014; Gulbransen et al. 2020; Lintzeris et al. 2020; Lucas and Walsh 2017; Sexton et al. 2016; Waissengrin et al. 2015). Symptom control is the primary reason for use of CBM, with most patients looking to address unalleviated symptoms, perceived symptom intensity, and burden on health-related quality of life independently of primary diagnosis (Sexton et al. 2016; Waissengrin et al. 2015; Baron et al. 2018; Purcell et al. 2019; Swift et al. 2005; Webb and Webb 2014). The Edmonton Symptom Assessment Scale-revised version (ESAS-r) is a validated scale to assess symptom burden developed for use in oncology and palliative care (Hui and Bruera 2017), it has relevance to medical cannabis care as patients are often treated for similar symptom management (Good et al. 2019; Pawasarat et al. 2020). Specifically, studies showed self-perceived improvement in ESAS-r emotional symptoms (anxiety and depression) scores following CBM treatment in oncology patients, while pain and wellbeing symptoms showed no improvement (Good et al. 2019; Pawasarat et al. 2020). Yet, RWE on CBD-rich products is scarce (Goodman et al. 2020; Shannon et al. 2019). In addition, although careful titration and treatment adjustment after initiation is critical to symptom improvement and adverse effects care, current literature has failed to address this issue.

In this study, we investigated treatment with CBD-rich products within a dedicated clinical setting in Quebec, Canada, and the effects on a very common clinical symptom expression of pain and comorbid anxiety and depression symptoms, as well as the effect on overall wellbeing. We also examined the relevant clinical effects that were observed when CBD-rich treatments were replaced by THC:CBD-balanced products at subsequent follow-up visits.

\section{Methods}

\section{Study population}

This study is a retrospective examination of patients who were prescribed CBD-rich products by physicians at a clinic dedicated to CBM treatments operating at four locations across Quebec, Canada. All data are collected as part of standard clinical procedures during the initial visit and during 3 (FUP1) and 6 (FUP2) month followup visits and extracted from electronic medical records (EMR) (Prosk et al., 2021). All data were anonymized following extraction from the EMR and no identifiers linking to original data were maintained. A waiver of consent was required and approved by Advarra Ethics Committee, who also approved the study protocol, and by the provincial privacy commission (La commission d'accès à l'information du Quebec).

Adult patients, at least 18 years of age, who were initially treated exclusively with CBD-rich products from 1 October 2017 to 31 May 2019 and for whom outcome scores and product information were recorded at FUP1 were included in this study. Patients were generally referred by primary-care physicians and specialists for an assessment on the suitability of medical cannabis to treat refractory symptoms. A complete medical history, 
including primary and secondary diagnoses, was collected at baseline visit. Medical cannabis treatment decisions are determined at the discretion of a clinic physician according to a standardized clinical procedure, including symptom identification, selection of product format, cannabinoid profile, and dosage based on existing evidence (MacCallum and Russo 2018; Cyr et al. 2018), but also to minimize risk of adverse effects. Patient and physician preference may also indicate initiation with products that have higher $\mathrm{CBD}$ and lower THC concentration in order to limit use of THC and its inherent potential adverse events. The follow-up visits serve to assess treatment compliance, safety, and effectiveness.

\section{CBD-rich products in Canada}

CBD-rich products are administered in various methods and formats, but most commonly as oral plant-derived extracts or oils and as inhaled dried flowers. In the Canadian medical cannabis program, CBD-rich cannabis oils contain approximately $0.5-1 \mathrm{mg}$ of $\mathrm{THC} / \mathrm{mL}$ and $20-25$ $\mathrm{mg}$ of $\mathrm{CBD} / \mathrm{mL}$ depending on the product manufacturer. Table 1 provides cannabinoid content and THC:CBD ratio for the three most common oil products (over 85\% of patients) authorized at the clinic. Furthermore, product details in this study sample are described in Table 3. The clinic procedure dictates that all products with a ratio of CBD (mg) to THC (mg) higher than 10 are considered CBD-rich products.

Treatment adjustments occur at follow-up visits as a result of lack of effectiveness, presentation of adverse effects, or social or economic barriers. Adjustments may include a change of the recommended CBD-rich product, method of administration, dosage, or a change in product formulation such as the introduction of THC: CBD-balanced or THC-rich products. We investigated the change from CBD-rich to THC:CBD products during FUP1 by forming two groups based on their product adjustment at FUP1 (CBD-rich vs THC:CBD). Products at FUP1 reflect those recommended at the visit. Therefore, the adjusted treatment affects only the evaluation at FUP2.

\section{Outcomes}

Patients age, sex, and diagnosis were recorded at baseline. Patients completed the ESAS-r (Edmonton Symptom Assessment System-revised version) at each visit. The ESAS-r is a self-administered scale, rating the severity of symptoms from 0 (absence of symptom) to 10 (worst possible severity) at the time of assessment (Hui and Bruera 2017). Symptoms evaluated include six physical- (pain, tiredness, nausea, drowsiness, lack of appetite, and shortness of breath), two emotional(depression, anxiety), and one overall wellbeing-related symptoms. ESAS scores can be categorized as mild (score 0 to 3 ) moderate (score 4 to 6) or high (score 7 and above) (Butt et al. 2008) and the threshold for clinically significant improvement is a decrease of 1 point (Hui et al. 2015). Since pain and mental health issues represent the most common symptoms for patients and physicians seeking medical cannabis treatments, we investigated effects on pain, depression, and anxiety symptoms as well as overall wellbeing. For each symptom, two groups of patients were formed: moderate-severe severity group in which a baseline score of 4 or more was recorded and a mild severity group with baseline score of 0 to 3 .

\section{Analyses}

Mean scores and standard deviation (SD), as well as percentage, where appropriate are presented for each variable. All analyses were performed on each ESAS-r symptom separately through the data analytics software $\mathrm{R}$ v4.0.2. An initial analysis compared the overall ESAS-r scores between each visit no matter the severity of the group, and looked at the role of product group (CBD/ THC:CBD vs CBD/CBD group) (between-factor). Tukey HSD post hoc test was used to confirm where the differences occurred between groups.

To determine whether CBD-based treatments have different effectiveness based on the severity of patient symptoms, two-way mixed ANOVAs with severity group as between-factor and visit as a within-factor were conducted to assess the change in ESAS-r scores between visits. Paired t-tests were subsequently performed to assess the difference in mean scores within each severity

Table 1 THC and CBD contents and associated THC:CBD ratio for the three most common oil products authorized at the clinic

\begin{tabular}{|c|c|c|c|c|c|c|c|c|c|}
\hline \multirow[b]{2}{*}{ Authorized dose range (in $\mathrm{ml} /$ intake) } & \multicolumn{3}{|c|}{ CBD-rich products at baseline } & \multicolumn{3}{|c|}{ THC:CBD-balanced products at FUP1 } & \multicolumn{3}{|c|}{ THC-rich products at FUP1 } \\
\hline & $0.1-2$ & & & $0.05-$ & & & $0.2-1$ & & \\
\hline Oil (mg/ml) & $\mathrm{THC}$ & $\mathrm{CBD}$ & Ratio THC:CBD & $\mathrm{THC}$ & CBD & Ratio THC:CBD & $\mathrm{THC}$ & $\mathrm{CBD}$ & Ratio THC:CBD \\
\hline Product 1 & 1.2 & 24 & $1: 25$ & 9.5 & 12 & $10: 13$ & 27.5 & $<1$ & $30: 1$ \\
\hline Product 2 & 1.3 & 30 & $1: 30$ & 15 & 15 & $15: 15$ & 18.5 & 0.7 & $20: 1$ \\
\hline Product 3 & $<1$ & 20 & $1: 20$ & 10 & 13.5 & $10: 13$ & 26.3 & $<1$ & $30: 1$ \\
\hline
\end{tabular}

The data is categorized by product category: CBD-rich products, THC:CBD-balanced products, and THC-rich products

$C B D$ cannabidiol, $T H C \triangle 9$-tetrahydrocannabinol, $S D$ standard deviation 
group between baseline and FUP1. Significant $p$ value was set at 0.05 and all analyses were two-tailed. Partial eta-squared $\left(\eta_{\mathrm{p}}^{2}\right)$ are reported to indicate magnitude of differences between groups.

\section{Results}

\section{General}

A total of 1095 patients were seen at the four clinic sites during the study period. Out of those, 715 were eligible for the study (at least 18 years old and initially treated exclusively with CBD-rich products). A total of 279 patients with ESAS-r scores and product information at FUP1 were analyzed (190 (68\%) female, mean age = 61.1, SD = 16.6). The analyzed sample did not differ from the study-eligible group in terms of age, sex, or THC and CBD initial doses (all $p \mathrm{~s}>0.4$ ). Table 2 outlines patient sample size and demographic information for each symptom and treatment group. Two hundred and ten $(75 \%)$ patients were prescribed CBD-rich products to treat chronic pain, 19 (7\%) for cancer-related symptoms, $21(7.5 \%)$ to treat neurological disorders (Parkinson's disease, multiple sclerosis, and drugresistant epilepsy among others), 8 patients for inflammatory disease (arthritis), 10 for gastrointestinal disorders (Chron's disease, inflammatory bowel syndrome, ulcerative colitis), 2 for anxiety, 1 for depression, 2 for headaches, and 6 unclassified. The chronic pain category included all medical indications for which pain was the main symptom such as but not limited to fibromyalgia, spinal stenosis, and chronic low back pain. Overall, 116 (41.6\%) patients adjusted their prescription by adding THC at FUP1 (either to a THC:CBD-balanced combination or a THC-rich treatment). Two hundred and three (73\%) patients had moderate/severe ESAS-r scores on at least 2 of the examined symptoms, 57 (20\%) on three, and $75(27 \%)$ on all four symptoms. Twenty-nine (10\%) patients report no moderate/severe symptoms; these people may use CBD for other ESAS-r symptoms not examined here (shortness of breath, tiredness, nausea, drowsiness, appetite). There was no statistical difference on either age, sex, or THC and CBD initial doses between the patients who completed one FUP versus those who completed two FUP (all $p$ s $>0.1$ ).

\section{CBD-rich products characteristics}

The baseline average daily doses for CBD and THC are presented in Table 3. The maximum initial CBD dose recorded $(156 \mathrm{mg})$ was prescribed for the treatment of pain of one patient. The maximum THC dose recorded at FUP1 $(90 \mathrm{mg}$ ) was prescribed for two patients for the treatment of pain.

\section{Outcome of CBD treatment}

Mean ESAS-r scores of pain, anxiety, depression symptoms, and overall wellbeing at baseline, FUP1, and FUP2 are described in Table 4 and Fig. 1.

All average ESAS-r scores decreased between baseline and FUP1 and FUP2. This was further demonstrated by ANOVAs which revealed a significant effect of visit on mean ESAS-r scores for each symptom assessed (pain: $\mathrm{F}(2,634)=4.9, p<0.008$; anxiety: $\mathrm{F}(2,624)=8.36, p<$ 0.001, depression: $\mathrm{F}(2,629)=5.36, p<0.004$; wellbeing: $\mathrm{F}(2,613)=8.31, p<0.001$; all $\eta_{\mathrm{p}}^{2}$ between 0.008 and 0.02 ). In all assessed symptoms, no significant main effect of adding THC at FUP1, nor visit-by-product interaction, were observed (all $p \mathrm{~s}>0.2$ ). Post hoc tests revealed ESAS-r mean scores significantly decreased between baseline and FUP1 (all ps $<0.003$ ) for all symptoms, between baseline and FUP2 for anxiety and wellbeing (both ps $<0.03$ ), but not between FUP1 and FUP2 for any symptoms (all ps $>0.5$ ). This suggests statistical improvement recorded at FUP1 is still present at FUP2 in all symptoms independently from treatment adjustment at FUP1.

\section{CBD treatment impact according to symptom severity}

From Table 2, moderate or severe scores at baseline were most common for pain (205 patients, $73.5 \%$ ) and poor wellbeing (202 patients, $72.4 \%$ ).

Clinical effect (difference of 1.3 to 2.5 points) observed in all symptoms for patients with moderate/severe symptoms between baseline and FUP1; however, there was no

Table 2 Demographic characteristics of 279 medical cannabis patients, by symptom group

\begin{tabular}{llll}
\hline & Sample size (percentage) & Number of female patients (percentage) & Mean age (SD) \\
\hline Overall sample & 279 & $190(68)$ & $61.1(16.6)$ \\
Moderate or severe pain symptom group & $205(73.5)$ & $150(73)$ & $61.8(15.9)$ \\
Moderate or severe anxiety symptom group & $138(48.5)$ & $97(70)$ & $61.43(16.3)$ \\
Moderate or severe depression symptom group & $115(41.2)$ & $81(70)$ & $60.5(15)$ \\
Moderate or severe wellbeing group & $202(72.4)$ & $141(70)$ & $60.8(16.1)$ \\
CBD/THC:CBD group & $116(41.6)$ & $75(65)$ & $60.38(14.4)$ \\
\hline
\end{tabular}

The symptom groups are mild and moderate or severe. The table presents the moderate or severe demographic characteristics. The CBD/THC:CBD group is composed of patients who added THC to their CBD-rich prescription during FUP1

$C B D$ cannabidiol, $T H C \triangle 9$-tetrahydrocannabinol, $S D$ standard deviation 
Table 3 Details of the THC and CBD component of the CBD-rich, the THC:CBD 1:1, and the THC-rich formulations

\begin{tabular}{|c|c|c|c|c|c|c|}
\hline & \multicolumn{2}{|c|}{$\begin{array}{l}\text { CBD-rich products at baseline }(n= \\
\text { 279) }\end{array}$} & \multicolumn{2}{|c|}{$\begin{array}{l}\text { THC:CBD-balanced products at FUP1 }(n= \\
104)\end{array}$} & \multicolumn{2}{|c|}{$\begin{array}{l}\text { THC-rich products at FUP1 }(n= \\
\text { 12) }\end{array}$} \\
\hline & THC & CBD & THC & CBD & THC & CBD \\
\hline Oil products (in mg/ml) & $0.1-2.0$ & $2.0-52.0$ & $0.6-30$ & $2.5-39$ & $1.25-45$ & $0-18$ \\
\hline Dried flower (in \% w/w) & 0.7 & 17.0 & $3.7-9$ & $7.7-13.4$ & $13-27$ & $0-0.5$ \\
\hline Average daily dose (mg) & 0.5 & 11.47 & 19.65 & 26.32 & 54.28 & 10.80 \\
\hline Standard deviation (mg) & 0.43 & 10.21 & 5.80 & 9.12 & 29.65 & 7.64 \\
\hline Maximum daily dose (mg) & 6 & 156 & 60 & 78 & 90 & 54 \\
\hline
\end{tabular}

Data comes from our sample of 279 patients

CBD cannabidiol, $T H C \triangle 9$-tetrahydrocannabinol

clinical effect for patients with mild symptoms (from 0.3 to - 1.8) (Fig. 2). No clinical effect was observed in any symptoms between FUP1 and FUP2 for patients with moderate/severe symptoms ( -0.4 to 0.5$)$ as well as for patients with mild symptoms (from -0.7 to 0.4 ).

\section{Pain}

The ANOVA revealed that all main and interaction effects were significant at the 0.001 level with effect sizes large for severity $\left(\eta_{p}^{2}=0.29\right)$, medium for visit $\left(\eta_{p}^{2}=\right.$ $0.06)$, and small for the interaction $\left(\eta_{p}^{2}=0.03\right)$. Post hoc tests revealed a significant score difference between baseline and FUP1 and FUP2 (both ps $<0.05$ ) but not between FUP1 and FUP2 $(p=0.98)$. Patients with moderate/severe symptoms on pain experienced important improvement at FUP1 $(\mathrm{t}(194)=7.61, p<0.001)$ whereas ESAS-r scores for patients with mild symptoms actually increased $(\mathrm{t}(64)=-2.03, p<0.05)$ (Fig. 2a).

\section{Anxiety}

There were significant effects of visit, severity group, and visit by group interaction (all $p s<0.001 ; \eta_{p}^{2}=0.006, \eta_{p}^{2}$ $=0.4, \eta_{\mathrm{p}}^{2}=0.1$, respectively). Post hoc tests revealed a significant score difference between baseline and FUP1 and FUP2 (both $p$ s $<0.001$ ) but not between FUP1 and FUP2 $(p=0.38)$. Although there was a large improvement for patients with moderate to severe anxiety symptoms $(\mathrm{t}(131)=9.36, p<0.001)$, the anxiety scores of

Table 4 Mean and standard deviation (SD) scores of ESAS-r scales for each severity group (mild or moderate/severe) and for each product group (CBD/CBD or CBD/THC:CBD)

\begin{tabular}{lllll}
\hline Mean (SD) & Pain & Anxiety & Depression & Wellbeing \\
\hline Baseline (sample size) & 277 & 270 & 272 & 268 \\
Overall sample & $5.14(2.57)$ & $3.86(3.19)$ & $3.16(3.08)$ & $5.34(2.61)$ \\
Mild severity group & $1.69(1.1)$ & $0.99(1.15)$ & $0.87(1.18)$ & $1.86(1.18)$ \\
Moderate or severe severity group & $6.34(1.7)$ & $6.61(1.78)$ & $6.3(1.86)$ & $6.47(1.83)$ \\
CBD/CBD group & $5.03(2.66)$ & $3.80(3.21)$ & $2.99(3.04)$ & $5.28(2.72)$ \\
CBD/THC:CBD group & $5.28(2.45)$ & $3.95(3.17)$ & $3.40(3.13)$ & $5.42(2.46)$ \\
FUP1 (sample size) & 262 & 261 & 261 & 254 \\
Overall Sample & $4.37(2.73)$ & $2.93(2.95)$ & $2.33(2.79)$ & $4.45(2.6)$ \\
Mild severity group & $2.3(2.4)$ & $1.62(2.08)$ & $4.12(1.78)$ & $3.73(2.75)$ \\
Moderate or severe severity group & $5.04(2.49)$ & $4.15(3.09)$ & $3.77(3.07)$ & $4.72(2.5)$ \\
CBD/CBD group & $4.09(2.67)$ & $2.74(2.87)$ & $2.23(2.71)$ & $4.43(2.6)$ \\
CBD/THC:CBD group & $4.75(2.78)$ & $3.2(3.05)$ & $2.47(2.9)$ & 102 \\
FUP2 (sample size) & 101 & 99 & $2.67(3.02)$ & $1.52(2.31)$ \\
Overall Sample & $4.7(2.7)$ & $2.85(3.01)$ & $1.32(1.89)$ & $3.63)$ \\
Mild severity group & $2.18(2.43)$ & $3.96(3.19)$ & $2.44(2.26)$ & $4.82)$
\end{tabular}

The CBD/THC:CBD group is composed of patients who added THC to their CBD-rich prescription during FUP1. ESAS-r scores varied between 0 and 10 for all assessed symptoms and all visits except for the anxiety scale at FUP2 for which the maximum score was 9 CBD cannabidiol, FUP1 follow-up visit at 3 month, FUP2 follow-up visit at 6 month, THC $\triangle 9$-tetrahydrocannabinol 


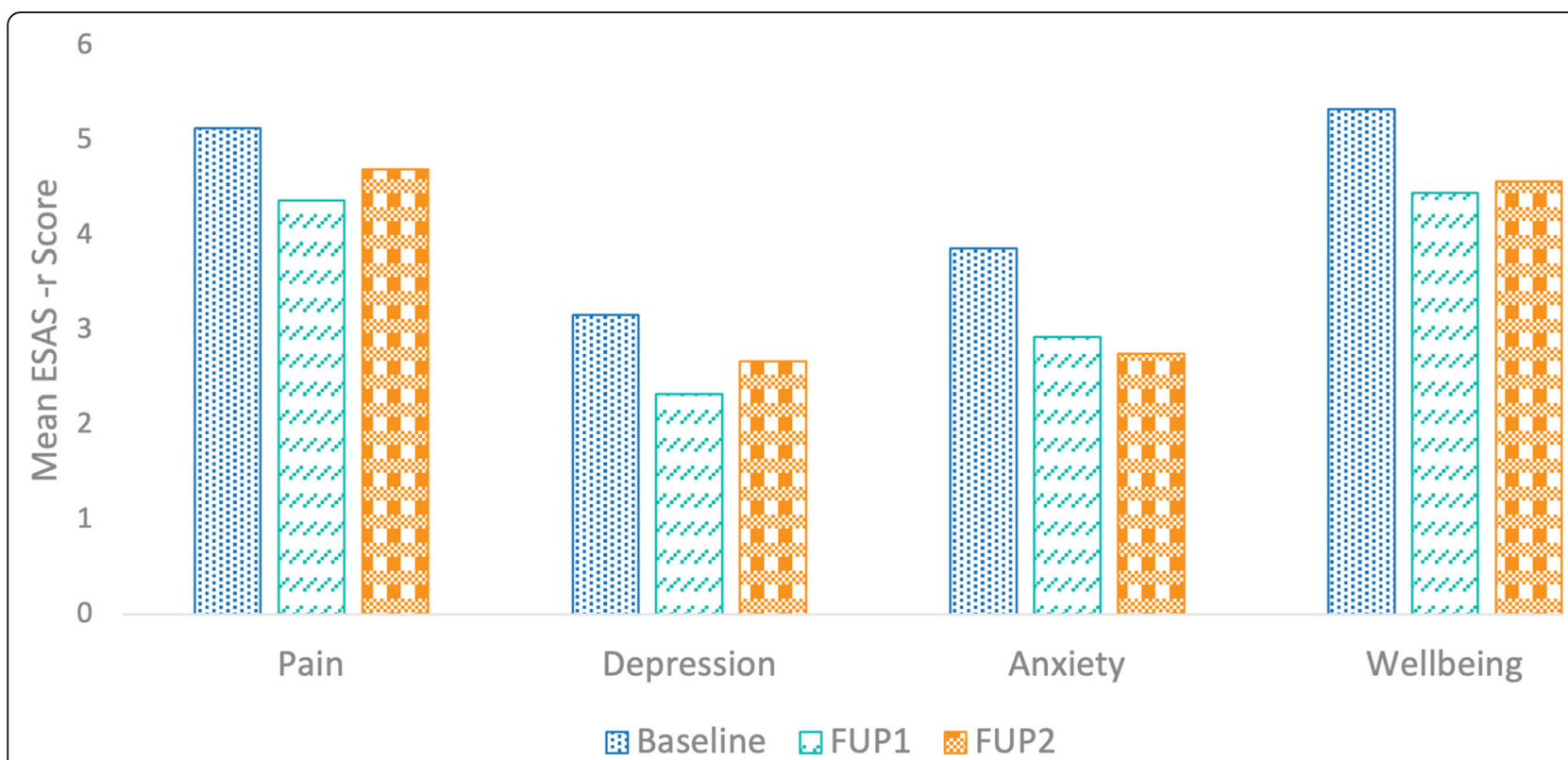

Fig. 1 CBD-rich treatment effectiveness on pain, anxiety, depression symptoms, and on overall wellbeing in 279 patients. FuP1, follow-up visit at 3 month; FUP2, follow-up visit at 6 month. Mixed ANOVAs revealed a significant effect of visit on symptom reduction between baseline and FUP1 but not between FUP1 and FUP2

patients with mild symptoms increased $(\mathrm{t}(119)=-3.19$, $p<0.01$ ) from baseline to FUP1 (Fig. 2b).

\section{Depression}

The ANOVA showed main effects of visit, severity group (both $p s<0.001$ with $\eta_{p}^{2}=0.04$ and $\eta_{p}^{2}=0.4$, respectively) and a significant group-by-visit interaction $(\mathrm{F}(2$, $\left.620)=34.47, p<0.001 ; \eta_{p}^{2}=0.1\right)$. Post hoc tests revealed a significant score difference between baseline and FUP1 and FUP2 (both $p$ s $<0.01$ ) but not between FUP1 and FUP2 $(p=0.85)$. Specifically, the scores of moderate/severe group decreased notably $(\mathrm{t}(110)=9.56$, $p<0.001$ ) between baseline and FUP1 but the scores of the group with mild depression symptoms did not $(p=$ 0.07) (Fig. 2c).

\section{Wellbeing}

The ANOVA showed main effects of visit, severity group (both $p s<0.001$ with $\eta_{p}^{2}=0.04$ and $\eta_{p}^{2}=0.3$ respectively) and a significant group-by-visit interaction $(\mathrm{F}(2$, $\left.597)=36.53, p<0.001 ; \eta_{p}^{2}=0.11\right)$. Post hoc tests revealed a significant main score difference between baseline and FUP1 and FUP2 (both $p$ s $<0.01$ ) but not between FUP1 and FUP2 $(p=0.89)$. Precisely, the scores of the group reporting good wellbeing increased $(\mathrm{t}(182)$ $=8.8, p<0.001)$ whereas scores of patients with worst wellbeing notably decreased $(\mathrm{t}(59)=-5.08, p<0.001)$ between FUP1 and FUP2 (Fig. 2d).

\section{Discussion}

This retrospective study explored the use of CBD-rich products in a medical cannabis clinical setting in Canada and associated effectiveness on a common symptom cluster presentation of pain, anxiety, depression, and poor sense of wellbeing, as measured by ESAS-r.

Patients treated with CBD-rich products were mainly women in their sixties, seeking predominantly chronic pain management.

Our findings show that overall effectiveness of CBD treatment is primarily by patients with moderate to severe symptoms. A deficiency in the endocannabinoid system (ECS) may provide a possible explanation for this result (Russo 2016). The ECS could be more deficient in patients with moderate/severe symptoms compared to mild symptoms leading to increased improvement in the first group. The absence of significant improvement for patients with mild symptoms at baseline may be explained by a smaller margin for symptom improvement. In such patients, CBD treatments may have been targeted to other clinical symptoms not assessed in the current study. There is a probable placebo effect; however, there were no differences in initial CBD doses between the severity groups. Furthermore, associated placebo effect would likely be decreased by FUP3M, also considering the significant treatment cost. The distinct beneficial impact of CBD treatment observed for patients with moderate-severe symptoms could elucidate discrepancies found in the literature. 


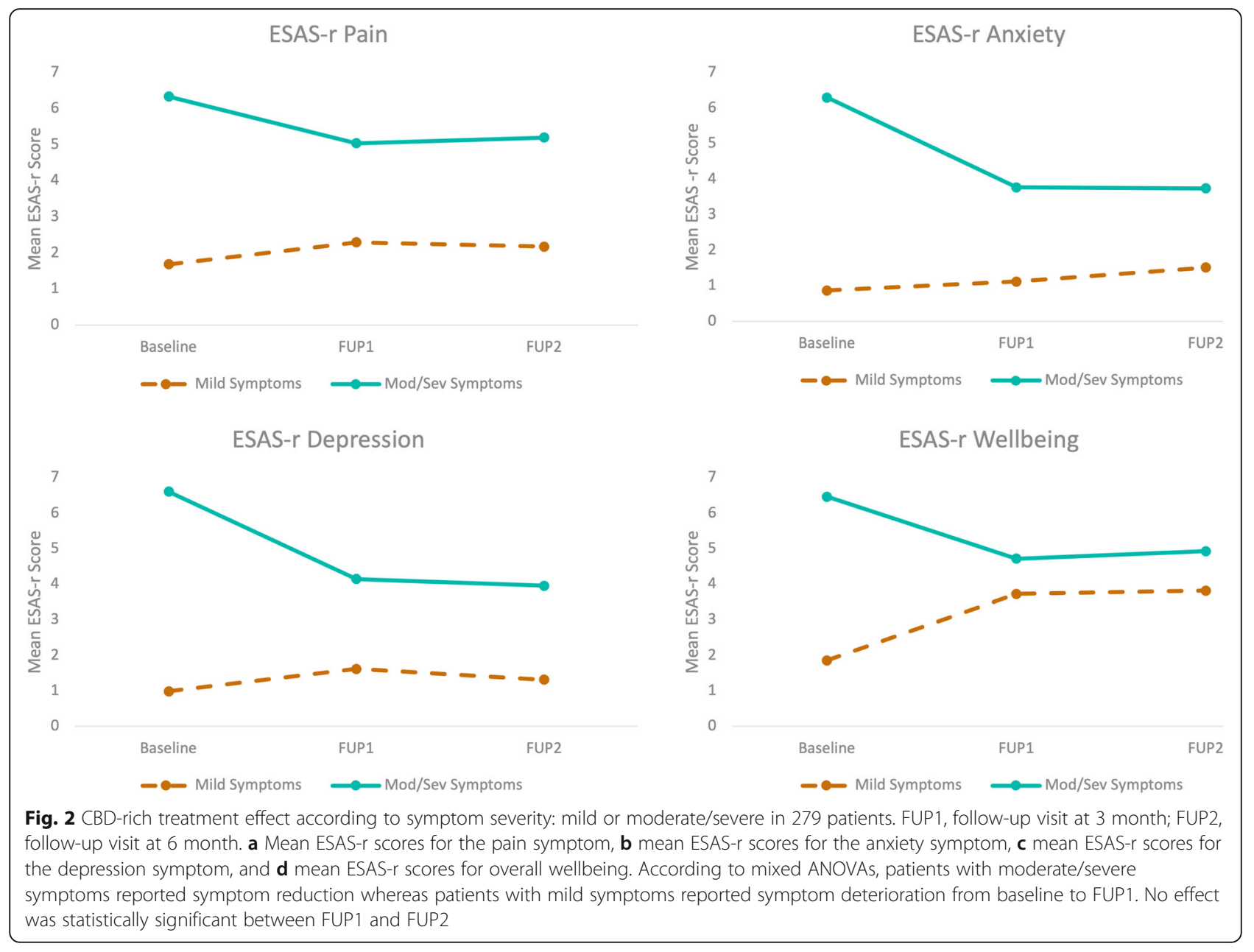

RCTs on CBM and pain symptoms provide inconclusive results; however, several report that treatments of THC and CBD have some benefit for pain management (Häuser et al. 2018; Russo 2008; Prosk et al. 2020). Our results are largely novel as research on the effect of CBD on pain control is very limited (Boyaji et al. 2020). The reduction in reported anxiety may also contribute to the improvement in pain perception.

Discrepancies still exist regarding the anxiolytic effect of CBD. Some RCTs indicate an anxiolytic effect of CBD upon experimentally induced scenarios (Bergamaschi et al. 2011; Zuardi et al. 2017; Bhattacharyya et al. 2010; Skelley et al. 2020); however, these findings are difficult to replicate (Larsen and Shahinas 2020; Hundal et al. 2018; Crippa et al. 2012). This reinforces our findings that CBD may have a differential effect depending on anxiety severity. Regarding the effects of CBD on depression symptoms, further research is required to draw conclusions (Khalsa et al. 2020; Schier et al. 2014; Turna et al. 2017).

The addition of THC to CBD during FUP1 did not produce any effect on ESAS-r scores at FUP2 in this analysis; however, the magnitude of the difference between groups is small. The examination of treatment regimen has been seldom addressed in the literature and further development is required to inform guidelines for prescription and refinement of clinical practice.

Furthermore, a significant discrepancy is observed between the recorded dosages of oral CBD in RCTs and dosages in real-world settings. The average daily CBD dosage authorized at our clinic $(11.5 \mathrm{mg})$ is closer to other observational studies (Gulbransen et al. 2020) compared to what is seen in RCTs (up to $1000 \mathrm{mg}$ for a single dose) (Larsen and Shahinas 2020). The presence of THC and other cannabinoids in CBD-rich products may affect the outcomes in this study. The majority of RCTs investigated single-dose administration of CBD making it difficult to compare observed treatment outcomes with chronic dosing clinical settings. Importantly, medical cannabis products are generally not covered by most insurers and patients rely on out-of-pocket payments. The cost of CBD remains very high globally, approximately \$CAD 5-20 per 100 mg (Canada Go 2021; Eisenstein 2019; Canada 2020). Availability of reliable 
cannabinoid testing in certain international jurisdictions is also limited. The gap between effective doses demonstrated in RCTs and the actual affordable doses demonstrated by RWE mandate the need for a precise pricing and marketing strategy at the initiation of any drug development process.

\section{Limitations}

Limitations are common in real-world data (RWD), especially in retrospective studies. In this study, with no control group, no causality effect can be drawn between CBD-rich treatment and symptom improvement. Most patients treated with CBM present with multiple severe symptoms and the analyses presented here are limited to identify the treatment outcomes for such patients. Further studies can investigate the use of CBD to treat several symptoms simultaneously.

The self-reported subjective assessment used may be biased by the patient's positive expectation of treatment, which could lead to a possible placebo effect. This perceived effectiveness bias may also be increased by social and economic barriers. The current context of medical cannabis access, including social stigma, high cost, and lack of universal insurance coverage can increase the patient selection bias. Self-selection bias is increased by the significant patient interest in medical cannabis as these patients must be motivated to access the non-traditional medication system. This bias limits the generalizability of results but is common across international medical cannabis regimens and should not discount the observed results. The heterogeneity of the patient population with a variety of diagnoses and the diversity of medical cannabis preparations also affects the external validity of the study. However, clinical findings from within Canada's controlled regulatory program do provide important models for international consideration. Future research is required in controlled clinical settings to examine these factors in order to provide a more complete account of CBD effectiveness.

Also, there was a large drop of sample size (53\% loss) due to missing data. Additionally, there was an important loss to follow-up at the 6-month visit (FUP2) due to missed appointment and cost barriers, limiting the power of the findings. The total treatment cost has significant impact on treatment continuation. Improved patient retention and more robust, harmonized data collection methods will improve future observational studies and allow for long-term assessment. Collection of detailed, accurate product information is a challenge, especially with inhaled products (Corroon et al. 2020). There are opportunities for administration devices and other technology advancements to improve this limitation. Lastly, this study did not include safety data assessment, future studies should investigate safety considerations of CBD (Chesney et al. 2020). Collection of high-quality RWD will require improvements in patient retention, data monitoring, and more robust data collection methods within a controlled clinical setting.

\section{Conclusion}

This study on CBD-rich products demonstrates the potential of RWE for the advancement of medical cannabis research and practice guidelines, especially in a world where $\mathrm{CBD}$ use is exponentially increasing but scientific data are limited. It revealed that CBD-rich treatments have a beneficial impact on patients with self-reported moderate or severe symptoms of pain, anxiety, or depression and overall wellbeing but not in patients with mild symptoms. Further investigation is clearly required, but as of now the hyped, and often illegal, marketed claims of CBD as a wellness product are unsubstantiated. Our findings have important and novel implications to clinical practice, especially the examination of treatment plan adjustment during the first follow-up after initiation with CBD treatments. Improvements in access regimes, oversight, and clarification from regulatory agencies are also needed to improve the validity of RWE and assessment of the use of CBD-rich products.

\section{Abbreviations}

CBD: Cannabidiol; CBM: Cannabinoid-based medicines; EMR: Electronic medical record; ESAS-r: Edmonton Symptom Assessment System-revised version; FUP: Follow-up visit; RWD: Real-world data; RWE: Real-world evidence; SD: Standard deviation; THC: $\Delta$ 9-Tetrahydrocannabinol

\section{Acknowledgements}

The authors would like to thank the participants to this study. The authors would like to acknowledge Santé Cannabis co-founder Dr. Michael Dworkind and key clinical leaders Dr. Antonio Vigano, Dr. Howard Mitnick, Dr. Alain Watier, and Youri Drozd, clinical data assistant, for his contribution to data technical help.

\section{Authors' contributions}

All authors contributed to conception and design, interpretation of data, manuscript writing, and final approval. LR and RG conducted the analysis of data. All authors agreed to be accountable for their own contributions. The author(s) read and approved the final manuscript.

\section{Funding}

This research was funded internally by Santé Cannabis clinic.

\section{Availability of data and materials}

The datasets used and/or analyzed during the current study are available from the corresponding author on reasonable request.

\section{Declarations}

\section{Ethics approval and consent to participate}

A waiver of consent was required and approved by Advarra Ethics Committee, who also approved the study protocol, and by the provincial privacy commission (La commission d'accès à l'information du Quebec).

\section{Consent for publication}

Not applicable.

\section{Competing interests}

L.R.: Clinical research associate, employee at Santé Cannabis. R.G.: Epidemiologic and statistic consultant for Santé Cannabis. 
C.EH.: Director of Research and Innovation, employee at Santé Cannabis. MF.A.: Associate Research Director of Santé Cannabis.

E.P.: President and co-founder of Santé Cannabis.

Santé Cannabis is a medical clinic, research, and training center dedicated to medical cannabis. The views expressed are those of the authors. This is a retrospective, observational study which took place at Santé Cannabis; therefore, the design and conduct of the study was executed by Santé Cannabis clinic staff. C.EH. and E.P. had a supporting role, in the retrospective protocol development. The authors had no role in the conduct of the study and collection of data. None of the authors are involved in the care of patients or in treatment decisions. The authors acted independently, and Santé Cannabis had no role in the analysis of the study, nor the writing of the manuscript or decision to publish. There is no financial gain for Santé Cannabis or for the authors to publish. The authors, while connected to Santé Cannabis, do not have a financial or professional incentive for the decision to publish.

Received: 15 January 2021 Accepted: 7 June 2021

Published online: 23 June 2021

\section{References}

Baron EP, Lucas P. Eades J, Hogue O. Patterns of medicinal cannabis use, strain analysis, and substitution effect among patients with migraine, headache, arthritis, and chronic pain in a medicinal cannabis cohort. J Headache Pain. 2018;19(1):37. https://doi.org/10.1186/s10194-018-0862-2.

Bergamaschi MM, Queiroz RHC, Chagas MHN, de Oliveira DCG, De Martinis BS, Kapczinski F, et al. Cannabidiol reduces the anxiety induced by simulated public speaking in treatment-naïve social phobia patients. Neuropsychopharmacology. 2011;36(6):1219-26. https://doi.org/10.1038/ npp.2011.6.

Bhattacharyya S, Morrison PD, Fusar-Poli P, Martin-Santos R, Borgwardt S, WintonBrown $T$, et al. Opposite effects of $\Delta$-9-tetrahydrocannabinol and cannabidiol on human brain function and psychopathology. Neuropsychopharmacology. 2010;35(3):764-74. https://doi.org/10.1038/npp.2009.184.

Bonn-Miller MO, Boden MT, Bucossi MM, Babson KA. Self-reported cannabis use characteristics, patterns and helpfulness among medical cannabis users. Am J Drug Alcohol Abuse. 2014;40(1):23-30. https://doi.org/10.3109/00952990.2 013.821477.

Boyaji S, Merkow J, Elman RNM, Kaye AD, Yong RJ, Urman RD. The role of cannabidiol (CBD) in chronic pain management: an assessment of current evidence. Curr Pain Headache Rep. 2020;24(2):4. https://doi.org/10.1007/s11 916-020-0835-4.

Butt Z, Wagner LI, Beaumont IL, Paice JA, Peterman AH, Shevrin D, et al. Use of a single-item screening tool to detect clinically significant fatigue, pain, distress, and anorexia in ambulatory cancer practice. J Pain Symptom Manage. 2008;35(1):20-30. https://doi.org/10.1016/j.jpainsymman.2007.02.040.

Canada H. Canadian cannabis survey 2020 summary [surveys; statistics]. 2020 [updated 2020-12-21]. Available from: https:/www.canada.ca/en/health-cana da/services/drugs-medication/cannabis/research-data/canadian-cannabissurvey-2020-summary.htm|\#a3.

Chesney E, Oliver D, Green A, Sovi S, Wilson J, Englund A, et al. Adverse effects of cannabidiol: a systematic review and meta-analysis of randomized clinical trials. Neuropsychopharmacology. 2020;45(11):1799-806. https://doi.org/10.1 038/s41386-020-0667-2.

Corroon J, Kight R. Regulatory status of cannabidiol in the United States: a perspective. Cannabis Cannabinoid Res. 2018;3(1):190-4. https://doi.org/10.1 089/can.2018.0030

Corroon J, MacKay D, Dolphin W. Labeling of cannabidiol products: a public health perspective. Cannabis Cannabinoid Res. 2020;5(4):274-8. https://doi. org/10.1089/can.2019.0101.

Martin-Santos R, Crippa JA, Batalla A, Bhattacharyya S, Atakan Z, Borgwardt S, et al. Acute effects of a single, oral dose of d9-tetrahydrocannabinol (THC) and cannabidiol (CBD) administration in healthy volunteers. Curr Pharm Des. 2012;18(32):4966-79. https://doi.org/10.2174/138161212802884780.

Crippa JAS, Hallak JEC, Zuardi AW, Guimarães FS, Tumas V, dos Santos RG. Is cannabidiol the ideal drug to treat non-motor Parkinson's disease symptoms? Eur Arch Psychiatry Clin Neurosci. 2019;269(1):121-33. https://doi. org/10.1007/s00406-019-00982-6.

Cyr C, Arboleda MF, Aggarwal SK, Balneaves LG, Daeninck P, Néron A, et al. Cannabis in palliative care: current challenges and practical recommendations. Ann Palliat Med. 2018;7(4):463-77-77-77. https://doi.org/1 0.21037/apm.2018.06.04.

Eisenstein M. The reality behind cannabidiol's medical hype. Nature. 2019; 572(7771):S2-4. https://doi.org/10.1038/d41586-019-02524-5.

Franco V, Bialer M, Perucca E. Cannabidiol in the treatment of epilepsy: current evidence and perspectives for further research. Neuropharmacology. 2021; 185:108442. https://doi.org/10.1016/j.neuropharm.2020.108442.

Freeman TP, Hindocha C, Green SF, Bloomfield MAP. Medicinal use of cannabis based products and cannabinoids. Bmj. 2019;365:11141. Published 2019 Apr 4. https://doi.org/10.1136/bmj.l1141.

Good PD, Greer RM, Huggett GE, Hardy JR. An open-label pilot study testing the feasibility of assessing total symptom burden in trials of cannabinoid medications in palliative care. J Palliat Med. 2020;23(5):650-5. https://doi. org/10.1089/jpm.2019.0540.

Goodman S, Wadsworth E, Schauer GL, Hammond D. Use and perceptions of cannabidiol products in Canada and in the United States. Cannabis Cannabinoid Res. 2020;0(0):null.

Governement of Canada. Licensed cultivators, processors and sellers of cannabis under the Cannabis Act 2021 [updated 2021-01-08]. Available from: https:/ www.canada.ca/en/health-canada/services/drugs-medication/cannabis/ industry-licensees-applicants/licensed-cultivators-processors-sellers.html. Accessed 4 Jan 2021.

Graham M, Lucas CJ, Schneider J, Martin JH, Hall W. Translational hurdles with cannabis medicines. Pharmacoepidemiol Drug Saf. 2020;29(10):1325-30. https://doi.org/10.1002/pds.4999.

Gulbransen G, Xu W, Arroll B. Cannabidiol prescription in clinical practice: an audit on the first 400 patients in New Zealand. BJGP Open. 2020;4(1): bjgpopen20X101010. https://doi.org/10.3399/bjgpopen20X101010.

Häuser W, Finn DP, Kalso E, Krcevski-Skvarc N, Kress H-G, Morlion B, et al. European Pain Federation (EFIC) position paper on appropriate use of cannabis-based medicines and medical cannabis for chronic pain management. Eur J Pain. 2018;22(9):1547-64. https://doi.org/10.1002/ejp.12 97.

Hui D, Bruera E. The Edmonton Symptom Assessment System 25 years later: past, present and future developments. J Pain Symptom Manage. 2017;53(3):63043. https://doi.org/10.1016/j.jpainsymman.2016.10.370.

Hui D, Shamieh O, Paiva CE, Perez-Cruz PE, Kwon JH, Muckaden MA, et al. Minimal clinically important differences in the Edmonton Symptom Assessment Scale in cancer patients: a prospective multicenter study. Cancer. 2015;121(17):3027-35. https://doi.org/10.1002/cncr.29437.

Hundal H, Lister R, Evans N, Antley A, Englund A, Murray RM, et al. The effects of cannabidiol on persecutory ideation and anxiety in a high trait paranoid group. J Psychopharmacol. 2018;32(3):276-82. https://doi.org/10.1177/02 69881117737400 .

Khalsa JH, Bunt G, Maggirwar SB, Kottilil S. COVID-19 and cannabidiol (CBD). J Addict Med. 2020;Publish Ahead of Print. https://doi.org/10.1097/ADM. 0000000000000771.

Larsen C, Shahinas J. Dosage, efficacy and safety of cannabidiol administration in adults: a systematic review of human trials. J Clin Med Res. 2020;12(3):129-41. https://doi.org/10.14740/jocmr4090.

Leehey MA, Liu Y, Hart F, Epstein C, Cook M, Sillau S, Klawitter J, Newman H, Sempio C, Forman L, Seeberger L, Klepitskaya O, Baud Z, Bainbridge J. Safety and tolerability of cannabidiol in Parkinson disease: an open label, doseescalation study. Cannabis Cannabinoid Res. 2020;5(4):326-36. https://doi. org/10.1089/can.2019.0068.

Lintzeris N, Mills L, Suraev A, Bravo M, Arkell T, Arnold JC, et al. Medical cannabis use in the Australian community following introduction of legal access: the 2018-2019 Online Cross-Sectional Cannabis as Medicine Survey (CAMS-18). Harm Reduct J. 2020;17(1):37. https://doi.org/10.1186/s12954-020-00377-0.

Lucas P, Walsh Z. Medical cannabis access, use, and substitution for prescription opioids and other substances: a survey of authorized medical cannabis patients. Int J Drug Policy. 2017;42:30-5. https://doi.org/10.1016/j.drugpo.201 7.01.011.

MacCallum CA, Russo EB. Practical considerations in medical cannabis administration and dosing. Eur J Intern Med. 2018;49:12-9. https://doi.org/1 0.1016/j.jjim.2018.01.004.

McGuire P, Robson P, Cubala WJ, Vasile D, Morrison PD, Barron R, et al. Cannabidiol (CBD) as an adjunctive therapy in schizophrenia: a multicenter randomized controlled trial. Am J Psychiatry. 2018;175(3):225-31. https://doi. org/10.1176/appi.ajp.2017.17030325. 
Millar SA, Stone NL, Bellman ZD, Yates AS, England TJ, O'Sullivan SE. A systematic review of cannabidiol dosing in clinical populations. Br J Clin Pharmacol. 2019;85(9):1888-900. https://doi.org/10.1111/bcp.14038.

Pawasarat IM, Schultz EM, Frisby JC, Mehta S, Angelo MA, Hardy SS, et al. The efficacy of medical marijuana in the treatment of cancer-related pain. J Palliat Med. 2020;23(6):809-16. https://doi.org/10.1089/jpm.2019.0374.

Prosk E, Arboleda MF, Rapin L, El Hage C, Dworkind M. The model of care at a leading medical cannabis clinic in Canada. Complement Ther Med. 2021;60: 102740. https://doi.org/10.1016/j.ctim.2021.102740.

Prosk E, Cyr C, Gamaoun R, El Hage C, Arboleda MF, Vigano A, editors. The CBD craze: making a case for observational clinical data to assess medical cannabis treatment effectiveness. Abstract accepted for the 30th Annual International Cannabinoid Research Society Symposium on the Cannabinoids. Galway; 2020. Cancelled due to COVID-19.

Prud'homme M, Cata R, Jutras-Aswad D. Cannabidiol as an intervention for addictive behaviors: a systematic review of the evidence. Subst Abuse: Res Treat. 2015;9:33-8. https://doi.org/10.4137/SART.S25081.

Purcell C, Davis A, Moolman N, Taylor SM. Reduction of benzodiazepine use in patients prescribed medical cannabis. Cannabis Cannabinoid Res. 2019;4(3): 214-8. https://doi.org/10.1089/can.2018.0020.

Russo EB. Cannabinoids in the management of difficult to treat pain. Ther Clin Risk Manag. 2008;4(1):245-59. https://doi.org/10.2147/TCRM.S1928.

Russo EB. Clinical endocannabinoid deficiency reconsidered: current research supports the theory in migraine, fibromyalgia, irritable bowel, and other treatment-resistant syndromes. Cannabis Cannabinoid Res. 2016; (1):154-65. https://doi.org/10.1089/can.2016.0009.

Russo EB. Cannabidiol claims and misconceptions. Trends Pharmacol Sci. 2017; 38(3):198-201. https://doi.org/10.1016/j.tips.2016.12.004.

Schier A, Ribeiro N, Coutinho D, Machado S, Arias-Carrion O, Crippa J, et al. Antidepressant-like and anxiolytic-like effects of cannabidiol: a chemical compound of Cannabis sativa. CNS Neurol Disord Drug Targets. 2014;13(6): 953-60. https://doi.org/10.2174/1871527313666140612114838.

Sexton M, Cuttler C, Finnell JS, Mischley LK. A cross-sectional survey of medical cannabis users: patterns of use and perceived efficacy. Cannabis Cannabinoid Res. 2016;1(1):131-8. https://doi.org/10.1089/can.2016.0007.

Shannon S, Lewis N, Lee H, Hughes S. Cannabidiol in anxiety and sleep: a large case series. Perm J. 2019;23:18-041. https://doi.org/10.7812/TPP/18-041.

Skelley JW, Deas CM, Curren Z, Ennis J. Use of cannabidiol in anxiety and anxietyrelated disorders. J Am Pharm Assoc. 2020;60(1):253-61. https://doi.org/10.1 016/j.japh.2019.11.008.

Swift W, Gates P, Dillon P. Survey of Australians using cannabis for medical purposes. Harm Reduct J. 2005;2(1):18. https://doi.org/10.1186/1477-7517-2-1 8.

Thiele E, Marsh E, Mazurkiewicz-Beldzinska M, Halford JJ, Gunning B, Devinsky O, et al. Cannabidiol in patients with Lennox-Gastaut syndrome: interim analysis of an open-label extension study. Epilepsia. 2019;60(3):419-28. https://doi. org/10.1111/epi.14670.

Turna J, Patterson B, Van Ameringen M. Is cannabis treatment for anxiety, mood, and related disorders ready for prime time? Depress Anxiety. 2017;34(11): 1006-17. https://doi.org/10.1002/da.22664.

Waissengrin B, Urban D, Leshem Y, Garty M, Wolf I. Patterns of use of medical cannabis among Israeli cancer patients: a single institution experience. J Pain Symptom Manage. 2015;49(2):223-30. https://doi.org/10.1016/j.jpainsymma n.2014.05.018.

Webb C, Webb S. Therapeutic benefits of cannabis a patient turvey. Hawai'i J Med Public Health. 2014;73(4):109-11.

Zuardi AW, Rodrigues NP, Silva AL, Bernardo SA, Hallak JE, Guimarães FS, et al. Inverted U-shaped dose-response curve of the anxiolytic effect of cannabidiol during public speaking in real life. Front Pharmacol. 2017;8:259. https://doi.org/10.3389/fphar.2017.00259.

\section{Publisher's Note}

Springer Nature remains neutral with regard to jurisdictional claims in published maps and institutional affiliations.

Ready to submit your research? Choose BMC and benefit from:

- fast, convenient online submission

- thorough peer review by experienced researchers in your field

- rapid publication on acceptance

- support for research data, including large and complex data types

- gold Open Access which fosters wider collaboration and increased citations

- maximum visibility for your research: over $100 \mathrm{M}$ website views per year

At BMC, research is always in progress.

Learn more biomedcentral.com/submissions 